\title{
Scaling laws in velocity-selective coherent-population-trapping laser cooling
}

\author{
G. Morigi,* B. Zambon, and N. Leinfellner ${ }^{\dagger}$ \\ Unità INFM, Dipartimento di Fisica, Università di Pisa, Piazza Torricelli 2, I-56126 Pisa, Italy \\ E. Arimondo \\ JILA, University of Colorado, Boulder, Colorado 80309-0440
}

(Received 1 November 1995)

\begin{abstract}
One-dimensional laser cooling based on velocity-selective coherent population trapping (VSCPT) has been investigated numerically through the solution of the optical Bloch equations and through a Monte Carlo analysis. The $1 \rightarrow 1$ and $2 \rightarrow 2$ transitions have been examined as a function of the atomic recoil frequency, the spontaneous-emission decay rate, and the Rabi frequency of the cooling laser. It has been found that for a large set of those parameters, the VSCPT cooling process may be described through scaling-law relations. The scaling laws are not valid at long atom-laser interaction times or large Rabi frequencies, where the atomic Doppler shift plays a significant role in the atomic motion evolution. Similar results for two atomic transitions suggest the validity of the scaling law for any one-dimensional VSCPT process.
\end{abstract}

PACS number(s): $32.80 . P \mathrm{j}$

\section{INTRODUCTION}

Velocity-selective coherent population trapping (VSCPT) allows laser cooling below the atomic recoil limit. VSCPT is based on the preparation of the atoms in a trapped coherent superposition of states with different internal variables and atomic momentum. VSCPT on the $J=1 \rightarrow J=1$ atomic transition has been applied for one-dimensional laser cooling of ${ }^{4} \mathrm{He}[1-4]$ and ${ }^{87} \mathrm{Rb}$ atoms [5] and for two- and threedimensional cooling of ${ }^{4} \mathrm{He}$ [6]. For extensions to atomic transitions with high- $J$ number, the difference in the kinetic energy of the states composing the coherent superposition should be compensated; otherwise the atomic superposition is not stable and only a very limited VSCPT cooling can be realized [7]. The kinetic-energy compensation can be produced either by an atomic energy level shift due to an applied static electric field [8] or by producing an atomic ac Stark shift $[9,10,12]$. Bichromatic VSCPT, produced by two laser fields at different frequencies, has been introduced as an alternative method to compensate for that kinetic-energy difference [11].

The Levy flight processes determine the atomic momentum distribution in a regime in which the interaction between the atom and the laser radiation is very long; for example, in helium a long-time interaction occurs over 100000 spontaneous emissions $[13,14]$. Those processes, having a very small but still significant probability of affecting the longtime atomic evolution, allow escape of the atoms from the trapped coherent superposition and limit the efficiency of the laser-cooling process. This decrease in efficiency may be compensated for by introducing boundaries limiting the maximum values of the atomic momentum, for instance, by

\footnotetext{
*Present address: Clarendon Laboratory, Oxford University, Oxford OX1 3PU, United Kingdom.

${ }^{\dagger}$ Permanent address: Institut für Experimentalphysik, Technische Universität, Petergasse 16, A-8010 Graz, Austria.

*Permanent address: Dipartimento di Fisica, Università di Pisa, I-56126 Pisa, Italy.
}

using an additional sub-Doppler cooling mechanism. The combination of VSCPT and polarization-gradient laser cooling has been explored by several authors [15-18,5].

The atomic preparation in a trapped atomic superposition requires the modification of the atomic momentum through a large number of absorption or emission photon processes. For heavier atoms, the modification in the atomic velocity produced by the photon momentum is smaller and very long interaction times are needed in order to produce the required cooling. The VSCPT theoretical analyses require numerical integration following the time evolution of the internal and external atomic variables. Different approaches such as solutions of the optical Bloch equations (OBEs), the quantum Monte Carlo (QMC) approach, and the band theory approach have been used for the investigation of the VSCPT cooling process. However, theoretical analyses are also faced with the problems of very long integration times and timeconsuming numerical integrations.

In this paper, the simplest VSCPT configuration of counterpropagating $\sigma^{+}, \sigma^{-}$laser fields is examined theoretically for scaling laws. We have explored the role of the different atom and laser parameters on the VSCPT process. We have defined the VSCPT efficiency through the fraction of atoms prepared in the trapped coherent superposition of states and we have determined the combination of the atom and laser parameters connecting the VSCPT efficiencies in different atomic systems. An indication of the proper parameters to be used for the scaling law has been derived from an analysis of the eigenvalues for the ground-state Hamiltonian obtained through an adiabatic elimination of the excited state. The scaling-law parameters have been confirmed by numerical simulations based either on OBEs or on the QMC approach.

Section II contains the definition of the atomic Hamiltonian and of the atomic basis used for the analysis. Section III introduces the effective ground-state Hamiltonian derived from the excited-state adiabatic elimination and its eigenvalues. The imaginary parts of those eigenvalues, i.e., the loss rates out of and into the trapped superposition, determine the time scale of the VSCPT process. Sections IV and V present results for the $1 \rightarrow 1$ and $2 \rightarrow 2$ transitions, respectively. In 

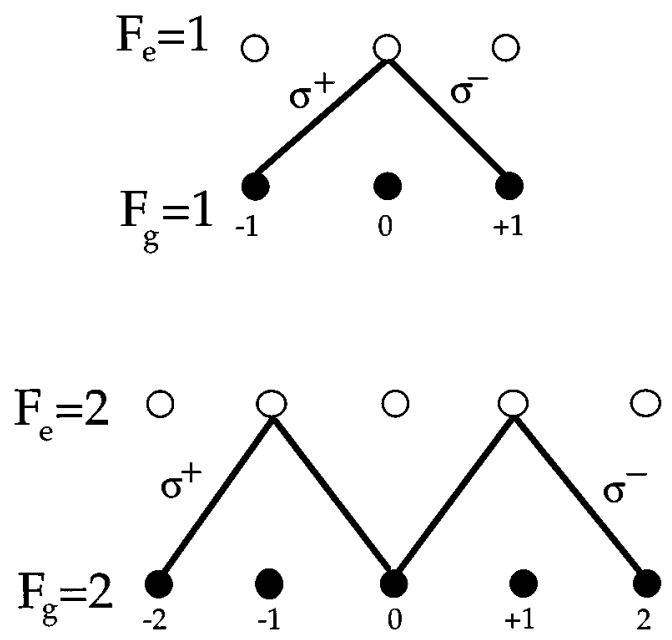

FIG. 1. Schematic representation of the $F_{g}=1 \rightarrow F_{e}=1$ and $F_{g}=2 \rightarrow F_{e}=2$ transitions considered in the present work for VSCPT under excitation by counterpropagating traveling-wave $\sigma^{+}$ and $\sigma^{-}$light beams. Only the relevant laser-driven transitions are reported.

Sec. VI we discuss the application of the scaling laws and their relation to other laser-cooling techniques.

\section{THEORETICAL DESCRIPTION}

The one-dimensional VSCPT laser cooling investigated here is based on the laser excitation of atoms either on the $F_{g}=1 \rightarrow F_{e}=1$ or $F_{g}=2 \rightarrow F_{e}=2$ transition, in which, in the absence of nuclear spin, as in ${ }^{4} \mathrm{He}$, the total angular momentum $F$ coincides with the electronic angular momentum $J$. The laser radiation is composed by two beams, one polarized $\sigma^{+}$and one polarized $\sigma^{-}$, that counterpropagate along the $\hat{\mathbf{z}}$ axis. For simplicity, the two laser beams are assumed to have the same intensity and the total laser electric field is given by

$$
E(z, t)=\frac{1}{2}\left[\hat{\boldsymbol{\epsilon}}_{+} \mathscr{E} e^{i\left(k z-\omega_{L} t\right)}+\text { c.c. }\right]+\frac{1}{2}\left[\hat{\boldsymbol{\epsilon}}_{-} \mathscr{E} e^{i\left(-k z-\omega_{L} t\right)}+\text { c.c. }\right],
$$

where $\hat{\boldsymbol{\epsilon}}_{+}$and $\hat{\boldsymbol{\epsilon}}_{-}$represent the unit vectors for the $\sigma^{+}$and $\sigma^{-}$waves, respectively. The wave-driven atomic transitions that are relevant for the VSCPT evolution are indicated in Fig. 1.

Both the internal and external atomic degrees of freedom must be quantized to study subrecoil laser cooling. Thus we use the states $|p ; F, m\rangle=|p\rangle \otimes|F, m\rangle$, where $|p\rangle$ is the eigenstate with eigenvalue $p$ of the atomic linear-momentum operator $\hat{P}$ along the $\hat{\mathbf{z}}$ propagation direction of the laser beams. The atomic Hamiltonian $H$ includes the kinetic and electronic energies $H_{\text {kin }}$ and $H_{\text {ele }}$ and the atom-laser interaction $V_{a l}$ :

$$
H=H_{\text {kin }}+H_{\text {ele }}+V_{a l} \text {. }
$$

For an atom with mass $M$ the kinetic part is

$$
H_{\text {kin }}=\frac{\hat{P}^{2}}{2 M}
$$

and kinetic energy will be expressed in terms of the recoil frequency $\omega_{R}$;

$$
\omega_{R}=\frac{\hbar k^{2}}{2 M}
$$

For the electronic energy the origin will be assumed at the energy of the $\left|F_{g}, m_{g}=0\right\rangle$ level. The excited states will be assumed to be always degenerate and to have energy $\hbar \omega_{0}$. The ground states will be assumed to be either degenerate, at zero energy, or split in energy because of an ac Stark light shift produced by an additional $\pi$-polarized laser beam on another $F_{g} \rightarrow F_{e}=F_{g}$ transition $[9,10]$. That light shift, $\hbar \omega_{\left|m_{g}\right|}^{\mathrm{LS}}$ for the $\left|F_{g}, m_{g}\right\rangle$ state, being produced by $\pi$-polarized light, depends on the $\left|m_{g}\right|$ value only and is equal to zero for the $\left|F_{g}, 0\right\rangle$ state. Thus the electronic Hamiltonian is written as

$$
\begin{aligned}
H_{\mathrm{ele}}= & \hbar \omega_{0} \sum_{m_{e}=-F_{e}}^{F_{e}}\left|F_{e}, m_{e}\right\rangle\left\langle F_{e}, m_{e}\right| \\
& +\sum_{m_{g}=1}^{F_{g}} \hbar \omega_{\left|m_{g}\right|}^{\mathrm{LS}}\left(\left|F_{g}, m_{g}\right\rangle\left\langle F_{g}, m_{g}\right|\right. \\
& \left.+\left|F_{g},-m_{g}\right\rangle\left\langle F_{g},-m_{g}\right|\right) .
\end{aligned}
$$

The atom-laser interaction depends on the ClebschGordan coefficients for the individual Zeeman transitions driven by the applied laser fields. Following the approaches of Ref. [19], the Rabi frequency will be defined on the basis of the reduced dipole moment $\left\langle F_{g}\|d\| F_{e}\right\rangle$, with the WignerEckart theorem for the dipole-moment matrix elements taken as

$$
\left\langle F_{g}, m_{g}\left|d_{i}\right| F_{e}, m_{e}\right\rangle=\frac{\left\langle F_{g}\|d\| F_{e}\right\rangle}{\left(2 F_{g}+1\right)^{1 / 2}}\left\langle F_{e}, m_{e} ; 1, i \mid F_{g}, m_{g}\right\rangle,
$$

with $i=0, \pm 1$. Thus the Rabi frequency $\Omega$ for the transition $F_{g} \rightarrow F_{e}$ associated with the $E$ electric field of both the $\sigma^{+}$ and $\sigma^{-}$waves is defined as

$$
\Omega=-\frac{\left\langle F_{g}\|d\| F_{e}\right\rangle \mathscr{E}}{\left(2 F_{g}+1\right)^{1 / 2} \hbar} .
$$

Through the use of this definition, the atom-laser interaction Hamiltonian is written

$$
\begin{aligned}
V_{a l}= & \frac{\hbar \Omega}{2} \sum_{p, m_{g}}\left[\left\langle F_{e}, m_{g}+1 ; 1,1 \mid F_{g}, m_{g}\right\rangle\left|p+1 ; F_{e}, m_{g}+1\right\rangle\right. \\
& \times\left\langle p ; F_{g}, m_{g}\right|+\left\langle F_{e}, m_{g}-1 ; 1,-1 \mid F_{g}, m_{g}\right\rangle \\
& \left.\times\left|p-1 ; F_{e}, m_{g}-1\right\rangle\left\langle p ; F_{g}, m_{g}\right|\right] e^{-i \omega_{L} t}+\text { H.c. }
\end{aligned}
$$

Equation (8) shows that only a closed set of states is coupled through the processes of absorption and stimulated emission. In Refs. [2,7], that closed set of states has been defined as the family $\mathscr{F}(q)$, where $q$ denotes the atomic momentum of the $\left|F_{e}, m_{e}=0\right\rangle$ state belonging to the closed set. Relevant families for the two atomic transitions here considered are 


$$
\begin{gathered}
\left|q-\hbar k ; F_{g}=1,-1\right\rangle, \quad\left|q ; F_{e}=1,0\right\rangle, \quad\left|q+\hbar k ; F_{g}=1,1\right\rangle ; \\
\left|q-2 \hbar k ; F_{g}=2,-2\right\rangle, \quad\left|q-\hbar k ; F_{e}=2,-1\right\rangle, \quad\left|q ; F_{g}=2,0\right\rangle, \\
\left|q+\hbar k ; F_{e}=2,1\right\rangle, \quad\left|q+2 \hbar k ; F_{g}=2,2\right\rangle . \quad \text { (9b) }
\end{gathered}
$$

Figure 1 reports only the laser-induced transitions between the states of the relevant families.

The final process to be included in the master equation for the atomic evolution is the spontaneous emission of the excited states with rate $\Gamma$. The basic roles of spontaneous emission are to produce a damping of the populations and coherences involving the excited state and to introduce an atomic redistribution among the families [2]. In spontaneous emission an atom, for instance, in the excited state $\left|q ; F_{e}=2, m_{e}=0\right\rangle$, emits a fluorescence photon arbitrarily directed in space, so that the atomic momentum $q$ along the quantization axis changes by any value between $-\hbar k$ and $\hbar k$. As a consequence, the ground states reached by the spontaneous-emission process belong to families $\mathscr{F}\left(q^{\prime}\right)$ with $q^{\prime} \neq q$. The term in the master equation describing redistribution among families has been reported in Ref. [2] for the case of the $F_{g}=1 \rightarrow F_{e}=1$ transition and the general expression for the repopulation of the ground state has been reported in Ref. [7]. Those relations will be used in our numerical analysis. It should be noticed also that, as in those references, the assumption of a uniform repopulation of the ground states by spontaneous emission will be introduced in our numerical calculations.

The atomic evolution of the population occupation for the atomic states has been determined through a numerical solution of the OBE or through the QMC analysis introduced by Mblmer, Castin, and Dalibard [20]. Because the OBE solution requires a very large basis for the atomic momentum in order to cope with the atomic diffusion toward large values, we have used as much as possible the QMC approach. For the $F_{g}=1 \rightarrow F_{e}=1$ transition, where an adiabatic elimination of the excited state could be applied, we used the quantumjump variation of the QMC approach introduced by $\mathrm{Wu}$, Holland, and Foot [12]. That quantum-jump variation produces a considerable gain in computational time with respect to the QMC method of Ref. [20]. In the case of the $F_{g}=2 \rightarrow F_{e}=2$ transition, the quantum-jump model proposed by Wu, Holland, and Foot [12] is not applicable for the interaction times and the Rabi frequencies considered in our analysis because it relies on approximate eigenvalues and eigenvectors not valid for our cases.

\section{GROUND-STATE LOSS RATES}

A convenient approach to analyzing the VSCPT features is the resolvent theory based on the adiabatic elimination of the excited state and the determination of the eigenvalues of a Hamiltonian acting on only the ground states. In the case of a small Rabi frequency $\Omega \ll\left|\Gamma / 2+\delta_{L}\right|$, where $\delta_{L}=\omega_{L}-\omega_{0}+\omega_{R}$ is the laser detuning, the action of the atom-laser interaction $V_{a l}$ on the ground states may be described through an effective Hamiltonian $H_{\text {res }}$ derived at the lowest order in a perturbation approach of the resolvent theory $[21]$ :

$$
H_{\mathrm{res}}=\tilde{V}_{a l} P_{e} \frac{1}{\hbar\left[\omega_{L}-E_{e}+E_{g}+i \Gamma / 2\right]} P_{e} \tilde{V}_{a l} .
$$

Here $P_{e}$ is the projector on the excited states and

$$
\tilde{V}_{a l}=e^{i P_{e} \omega_{L} t} V_{a l} e^{-i P_{e} \omega_{L} t} .
$$

In the denominator of Eq. (10) the energies $E_{e}$ and $E_{g}$ of the excited and ground states will include also the kineticenergy part. Thus the present derivation of the loss rates is more precise than the one reported in Ref. [7], because, as in Ref. [22], the Doppler shift contribution $p k / M$ to the laser detuning is included in the denominator of the resolvent expression. The following effective Hamiltonian $H_{\text {eff }}$ is now acting on the ground state:

$$
H_{\text {eff }}=H_{\text {kin }}+H_{\text {ele }}+H_{\text {res }} \text {. }
$$

Because photon absorption from the ground state is equivalent to a loss rate, the Hamiltonian $H_{\text {eff }}$ acting on the ground state is complex. The real and imaginary parts of its eigenvalues are the eigenenergies and loss rates for the ground eigenstates, respectively, as examined in Refs. [22, 7, 23]. Diagonalization of the Hamiltonian $H_{\text {res }}$ on the ground states belonging to the family $\mathscr{F}(q)$ allows us to derive the loss rate for the eigenstates.

A VSCPT perfect-trap ground state has zero loss rate and is stable against both the atom-laser interaction and the kinetic energy coupling to other states. Ground states with a loss rate not exactly zero, but small, could also be used to realize VSCPT laser cooling, even if with a smaller efficiency. Those states with nonzero loss rates have been initially considered in Ref. [7] and denoted as leaky states in Ref. [10]. An atomic configuration in which the leakiness of the states is compensated for by a modification of the atomic Hamiltonian, for instance, through the light shifts $\omega_{|m|}^{\mathrm{LS}}$ of Eq. (2), is denoted nonleaky.

$$
\text { IV. } F_{g}=1 \rightarrow F_{e}=1 \text { TRANSITION }
$$

\section{A. Eigenvalues}

The ground-state eigenvalues are obtained by diagonalizing the effective Hamiltonian $H_{\text {eff }}$ of Eq. (12) on the two ground states of the family $\mathscr{F}(q)$ of Eq. (9a). By eliminating common kinetic-energy terms through a phase change for the states, the $2 \times 2$ matrix of the effective Hamiltonian is

$$
H_{\mathrm{eff}}(q)=\hbar\left(\begin{array}{cc}
-\frac{k q}{M}+R_{+} & R_{-} \\
R_{+} & +\frac{k q}{M}+R_{-}
\end{array}\right),
$$

where the $R_{ \pm}$level-shift operators defined in the resolvent theory of Ref. [21] are

$$
R_{ \pm}=\frac{1}{4} \frac{\Omega^{2} / 2}{\delta_{L} \pm k q / M+i \Gamma / 2} .
$$

For $\delta_{L}=0$ the $E_{+}(q)$ and $E_{-}(q)$ eigenvalues are 


$$
\begin{aligned}
\frac{E_{+}(q)}{\hbar}= & -i \frac{\Gamma^{\prime}}{1+\left(q / p_{e}\right)^{2}}\left[1+\left(1-\left(\frac{q}{\alpha \hbar k}\right)^{2}\left\{1+2\left(\frac{q}{p_{e}}\right)^{2}\right.\right.\right. \\
& \left.\left.\left.+\left(\frac{q}{p_{e}}\right)^{4}+\frac{\alpha \hbar k}{2 p_{e}}\left[1+\left(\frac{q}{p_{e}}\right)^{2}\right]\right\}\right)^{1 / 2}\right], \\
\frac{E_{-}(q)}{\hbar}= & -i \frac{\Gamma^{\prime}}{1+\left(q / p_{e}\right)^{2}}\left[1-\left(1-\left(\frac{q}{\alpha \hbar k}\right)^{2}\left\{1+2\left(\frac{q}{p_{e}}\right)^{2}\right.\right.\right. \\
& \left.\left.\left.+\left(\frac{q}{p_{e}}\right)^{4}+\frac{\alpha \hbar k}{2 p_{e}}\left[1+\left(\frac{q}{p_{e}}\right)^{2}\right]\right\}\right)^{1 / 2}\right] .
\end{aligned}
$$

Here we have introduced (i) the pumping rate $\Gamma^{\prime}$ at resonance $\delta_{L}=0$,

$$
\Gamma^{\prime}=\frac{\Omega^{2}}{2 \Gamma}
$$

(ii) an adimensional parameter $\alpha$ proportional to the ratio between the pumping rate $\Gamma^{\prime}$ and the recoil frequency $\omega_{R}$,

$$
\alpha=\frac{\Omega^{2}}{8 \Gamma \omega_{R}},
$$

and (iii) the momentum $p_{e}$ for which the atomic Doppler shift is equal to the natural linewidth

$$
\frac{k p_{e}}{M}=\frac{\Gamma}{2} .
$$

As shown in Ref. [13] the parameter $p_{e}$ defines also the range of the Doppler force acting in the VSCPT scheme. Our pumping rate $\Gamma^{\prime}$ is two times smaller than the one defined in Refs. [22] and [16] because it is defined for the Schrödinger states and not for the density matrix elements as in those references. The following relation between $\Gamma^{\prime}$ and $\alpha$ exists:

$$
\Gamma^{\prime}=4 \alpha \omega_{R} .
$$

At $|q| \ll p_{e}$ and $q^{2} \ll \alpha \hbar k p_{e}$, the imaginary parts of Eqs. (15a) and (15b), i.e., the ground-state loss rates, are for $|q| \leqslant \hbar k \alpha$,

$$
\begin{aligned}
& \Gamma_{C}(q)=\Gamma^{\prime}\left\{1+\left[1-\left(\frac{q}{\hbar k \alpha}\right)^{2}\right]^{1 / 2}\right\}, \\
& \Gamma_{\mathrm{NC}}(q)=\Gamma^{\prime}\left\{1-\left[1-\left(\frac{q}{\hbar k \alpha}\right)^{2}\right]^{1 / 2}\right\},
\end{aligned}
$$

and for $|q| \geqslant \hbar k \alpha$,

$$
\Gamma_{\mathrm{NC}}(q)=\Gamma_{C}(q)=\Gamma^{\prime} .
$$

The labels $C$ and NC, coupled and noncoupled, refer to the two following linear superposition of ground states within the $\mathscr{F}(q)$ family:

$$
\left|\mathscr{S}_{C}(q)\right\rangle=\frac{1}{\sqrt{2}}\left(\left|q-\hbar k ; F_{g}=1,-1\right\rangle+\left|q+\hbar k ; F_{g}=1,1\right\rangle\right),
$$
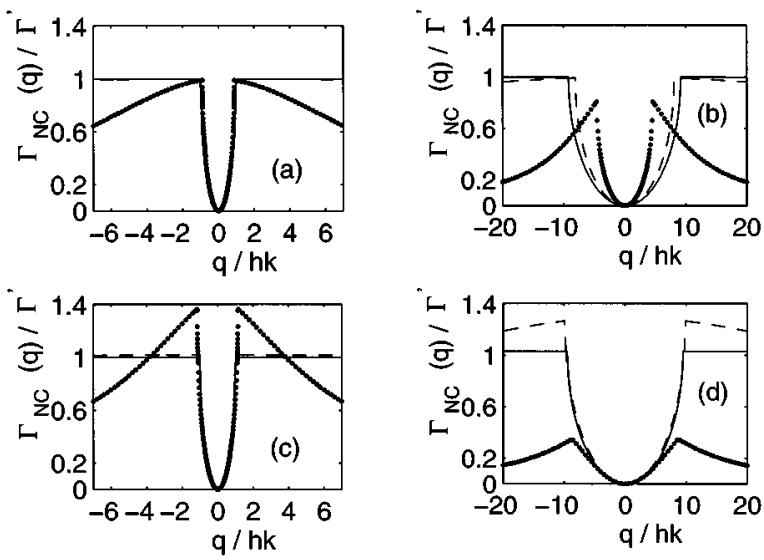

FIG. 2. Plot of the loss rate $\Gamma_{\mathrm{NC}}(p) / \Gamma^{\prime}$ vs $p / \hbar k$ for the $1 \rightarrow 1$ transition: (a) and (c) $\alpha=1$ and (b) and (d) $\alpha=9$. The loss rates in (a) and (c) have been obtained by diagonalizing the effective Hamiltonian acting on the ground states, those in (b) and (d) by diagonalizing the total Hamiltonian. Dots refer to helium, dashed lines to sodium, and solid lines to cesium.

$$
\left|\mathscr{S}_{\mathrm{NC}}(q)\right\rangle=\frac{1}{\sqrt{2}}\left(\left|q-\hbar k ; F_{g}=1,-1\right\rangle-\left|q+\hbar k ; F_{g}=1,1\right\rangle\right) \text {. }
$$

Equation (20b) shows that state $\left|\mathscr{J}_{\text {NC }}(q=0)\right\rangle$ is a perfecttrap ground state with zero loss rate. The loss rates of Eqs. (20a) and (20b) depend on the parameters $\Gamma^{\prime}$ and $\alpha$ and these parameters describe completely the VSCPT evolution, independently of the considered atom. For a given atom, i.e., for a given recoil frequency $\omega_{R}, \Gamma^{\prime}$ and $\alpha$ are not independent, but connected by Eq. (19). Equations (20a) and (20b) are not exact: they represent an approximation to the eigenvalues of Eqs. (15a) and (15b) that is valid in the limit $p_{e} \rightarrow \infty$, i.e., in the case in which the Doppler shift has no influence on the VSCPT evolution. Results for the loss rate $\Gamma_{\mathrm{NC}}(q)$, in units of $\Gamma^{\prime}$, as a function of the $q$ label of the family are reported in Figs. 2(a) and 2(b) for the cases of $\alpha=1$ and 9. The data in these figures refer to (i) helium atoms in the triplet metastable state excited by 1080 -nm radiation, as in the experiments of Refs. [1, 3, 4, 6], (ii) sodium atoms excited by the resonant 589-nm radiation, and (iii) cesium atoms irradiated by resonant $852-\mathrm{nm}$ radiation on an imaginary $F_{g}=1 \rightarrow F_{e}=1$ transition. The $\alpha$ values have been chosen in order to produce an efficient VSCPT cooling within a reasonable interaction time, as discussed in Sec. IV B. The parameter $\alpha=1$ corresponds to the following Rabi frequencies: for helium, $\Omega=0.46 \Gamma$; for sodium, $\Omega=0.14 \Gamma$; and for cesium, $\Omega=0.055 \Gamma$.

The plots of Fig. 2 show that, for a given $\alpha$ value and with the scaling law applied to $\Gamma_{\mathrm{NC}}$, the loss rates have the same functional dependence on $q$. In particular the region around $q=0$, or more precisely the Raman hole for $|q| \leqslant \alpha \hbar k$, is equal for all the atoms. As discussed in Ref. [16], the Raman hole region determines the atoms trapped by VSCPT cooling, as well as the efficiency of the process. A difference between the loss rates arises at large- $q$ momentum values, for $q \sim p_{e}$, where the atomic Doppler shift becomes relevant for the 
atomic evolution. From the atomic transition parameters we calculate

$$
p_{e}^{\mathrm{He}} \approx 9.4 \hbar k^{\mathrm{He}}, \quad p_{e}^{\mathrm{Na}} \approx 100 \hbar k^{\mathrm{Na}}, \quad p_{e}^{\mathrm{Cs}} \approx 570 \hbar k^{\mathrm{Cs}} .
$$

On the plots of Fig. 2 the decrease in the $\Gamma_{\mathrm{NC}}(q)$ loss rate may be observed for helium at $|q| \sim p_{e}^{\mathrm{He}}$.

In order to test the validity limits of the resolvent approach, the loss rates have been derived also from the diagonalization of the Hamiltonian $H$ of Eq. (2) within the family $\mathscr{F}(q)$ and the results are reported in Figs. 2(c) and 2(d). It appears that for sodium and cesium, the loss rates are nearly equal in the resolvent approach and in the approach based on the full Hamiltonian. For helium, as a consequence of the large recoil frequency $\omega_{R}$, a given $\alpha$ value corresponds to a large Rabi frequency and the validity of the resolvent approach is scarse even for $\alpha=1$.

\section{B. Trapped fraction}

Through numerical solution of either OBE or QMC evolution we have calculated the efficiency of the VSCPT cooling process as a function of the atom and laser parameters and of the interaction time $\Theta$ between the atom and laser. With increasing interaction time, the atoms are concentrated into the $\left|\mathscr{S}_{\mathrm{NC}}(q)\right\rangle$ states of Eq. (22b) with $q \sim 0$. A measurement of the atomic momentum on those states will provide a momentum $p$ around $\pm \hbar k$ with a spread $\delta p$ decreasing as $\Theta^{-1 / 2}$; see Ref. [2]. The efficiency of the VSCPT process is measured by the fraction of atoms that are trapped in states $\left|\mathscr{S}_{\mathrm{NC}}(q \sim 0)\right\rangle$ and have atomic momentum $p \sim \pm \hbar k$. The definition of the trapping efficiency is quite arbitrary. Following Ref. [13], we will consider the trapped fraction $f$ as composed by the fraction of atoms in states $\left|\mathscr{S}_{\mathrm{NC}}(q)\right\rangle$ with $|q|<\hbar k / 10$. QMC results for $f$ versus the interaction time $\Theta$ are reported in Fig. 3 for $\alpha=1$ and 9. The initial atomic momentum distribution was assumed to be Gaussian with a standard deviation $\delta p_{0}=\hbar k$, even if such a narrow initial distribution cannot be realized through a laser-cooling process. The dependence of the trapped fraction on the initial momentum standard deviation is discussed next.

For a generic atomic state vector expressed as a linear combination of the eigenstates with eigenvalues $E_{+}(q)$ and $E_{-}(q)$, the slow time evolution occurs on the time scale of the smaller term between $\hbar / E_{+}(q)$ and $\hbar / E_{-}(q)$. Equations (15a) and (15b) show that, apart from the noncoupled states within the Raman hole, the time evolution takes place on the $\left(\Gamma^{\prime}\right)^{-1}$ time scale. Thus for the trapped fraction of Fig. 3, the interaction time $\Theta$ has been measured in $\left(\Gamma^{\prime}\right)^{-1}$ units. It may be noticed that by choosing for different atoms a different Rabi frequency $\Omega$ in order to realize the same $\alpha$ value and by measuring the time on the $\Gamma^{\prime}$ scale, the same functional dependence of $f$ versus $\Theta$ is obtained at whichever atomic parameter. In terms of real-time units, the full time scale of Fig. 3 varies from $4 \times 10^{3} \Gamma^{-1}$ for helium to $3 \times 10^{5} \Gamma^{-1}$ for cesium: in a heavy atom a longer interaction time is required for realizing the same trapped fraction. In effect, at a fixed $\alpha$ value, Eq. (19) shows that the $\Gamma^{\prime-1}$ pumping time scale, or the number of scattered spontaneous-emission photons, is proportional to $\omega_{R}^{-1}$. For a heavy atom with large $\omega_{R}^{-1}$, the required pumping time $\Gamma^{\prime-1}$ is longer. In order to use the scaling law to estimate the efficiency of a generic VSCPT
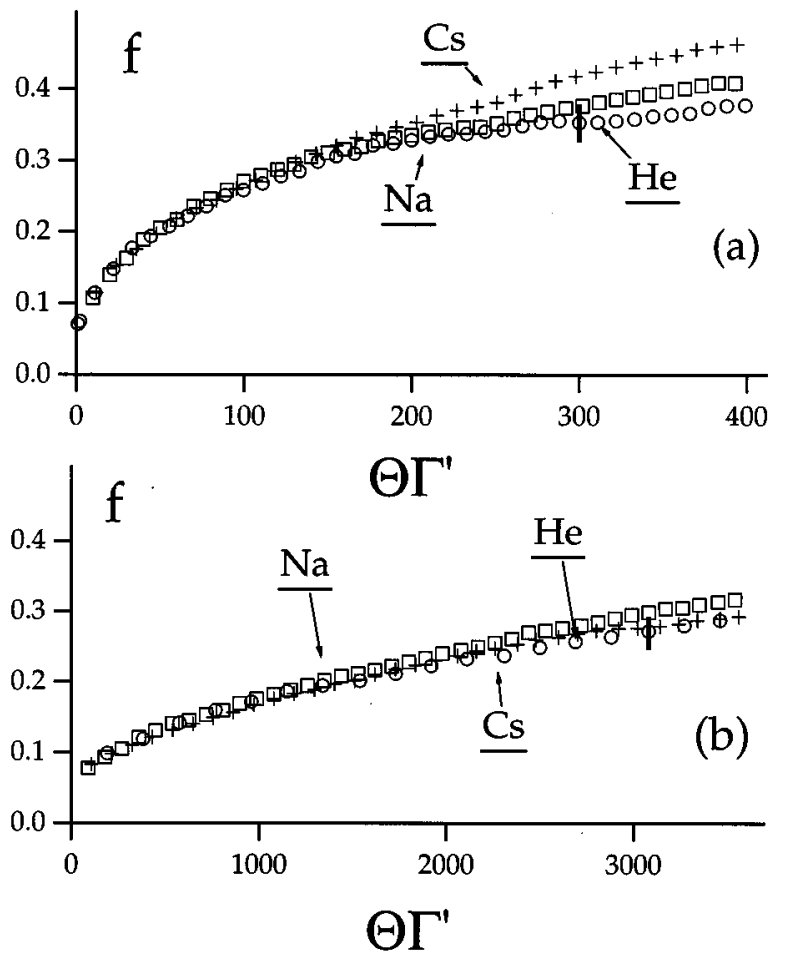

FIG. 3. QMC results for the fraction of trapped atoms vs the interaction time $\Theta \Gamma^{\prime}$ for a $1 \rightarrow 1$ transition, using the parameters of helium, sodium, and cesium atoms, at (a) $\alpha=1$ and (b) $\alpha=9$. The vertical error bar defines the typical QMC indetermination. Note that the trapped fraction does not depend on the atomic parameters.

trapping process, a numerical solution based on OBEs or the QMC approach may be performed on an atomic system having a shorter interaction time (for instance, helium) and the result properly scaled to the required atom.

We have noticed some deviations from the scaling law, mainly at large- $\alpha$ values. Those differences are a consequence of the Doppler shift modification in the functional dependence for the loss rates $\Gamma_{\mathrm{NC}}(q)$ versus $q$. Figure 2(a) at $\alpha=9$ shows that because of that modification, the helium loss rate presents a sharp maximum. Thus we have investigated whether a better agreement of the results for different atoms could be obtained by scaling the interaction time with the maximum value of $\Gamma_{\mathrm{NC}}(q)$, instead of $\Gamma^{\prime}$. The indetermination in our QMC results, reported as a vertical bar in Fig. 3, has not allowed us to reach a definitive conclusion on the validity of such refined time scaling.

The Doppler shift, breaking down the $\Gamma_{\mathrm{NC}}(q)$ scaling law at large values of $q$, modifies the atomic evolution at large values of the interaction time $\Theta$. This phenomenon has been investigated by Bardou et al. [13] within the Levy flight statistical approach. For a long interaction time, the atoms having a small rate for escape from the noncoupled state $\left|\mathscr{S}_{\text {NC }}(q \sim 0)\right\rangle$ acquire a finite probability of momentum diffusion toward large momentum values. Thus they are lost for the trapping process because they present a large Doppler shift and cannot be excited any longer by the laser radiation. As a consequence, at $\Theta \rightarrow \infty$, the fraction $f$ reduces to zero. In the long-time evolution where the Doppler shift is important, the scaling law for the fraction $f$ is not valid. The range of 


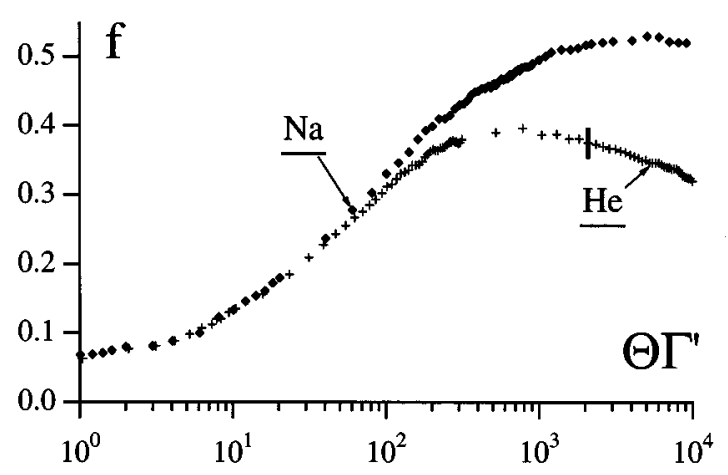

FIG. 4. QMC results for the fraction of trapped atoms vs the interaction time $\Theta=\Gamma^{\prime}$ at $\alpha=1$ for the $1 \rightarrow 1$ transition in helium and sodium. The vertical error bar defines the typical QMC indetermination. At interaction times $\Theta \geqslant \Theta_{e}^{\mathrm{He}}$ the efficiency of the VSCPT process depends on the atomic species.

validity of the scaling law for the loss rate $\Gamma_{\mathrm{NC}}(q)$, determined by $p_{e}$, allows us to derive the time limitation to the scaling law for the trapped fraction. In the case of an atomic momentum initial distribution having width $\delta p_{0} \ll p_{e}$, we introduce the following interaction time $\Theta_{e}$ associated to $p_{e}$, as in Ref. [13]:

$$
\Gamma^{\prime} \Theta_{e}=\left(\frac{p_{e}}{\hbar k}\right)^{2}
$$

$\Theta_{e}$ defines a Doppler time scale: for an interaction time $\Theta \ll \Theta_{e}$ the Doppler shift is small and the laser frequency remains within the natural linewidth of the atomic absorption line. At times $\Theta \gg \Theta_{e}$ the diffusion of the atoms in the momentum space could bring them to large values of atomic momentum in which the Doppler shift is relevant and the laser excitation does not occur. Thus the scaling law for the trapped fraction ceases its validity at $\Theta \sim \Theta_{e}$. It should be pointed out that the definition of Eq. (24) is meaningful only if $\alpha \hbar k \ll p_{e}$, so that the absorption line is not saturation broadened. By using the $p_{e}$ values from Eq. (23) for the different atoms, we derive the following interaction times:

$$
\Gamma^{\prime} \Theta_{e}^{\mathrm{He}} \approx 100, \quad \Gamma^{\prime} \Theta_{e}^{\mathrm{Na}} \approx 10, \quad \Gamma^{\prime} \Theta_{e}^{\mathrm{Cs}} \approx 3 \times 10^{4} .
$$

For a VSCPT comparison between different atoms, the scaling law may be applied for times up to the $\Theta_{e}$ value of the lighter atom used as a reference. Thus, for a determination of the cesium trapping efficiency at very long interaction times, a calculation on sodium provides a better approximation than one on helium.

Figure 4 reports QMC results for the trapped fraction as a function $\Theta$, at $\alpha=1$ and at $\Theta$ values longer than those of Fig. 3. Helium and sodium atoms are considered. At times $\Theta_{e}^{\mathrm{He}}$ $\ll \Theta \ll \Theta_{e}^{\mathrm{Na}}$, the trapped fraction for helium decreases, whereas the sodium trapped fraction continues to increase. In Refs. $[13,24]$ it was shown that, neglecting Doppler shift, the trapped fraction increases toward the limiting value $f_{0}$ :

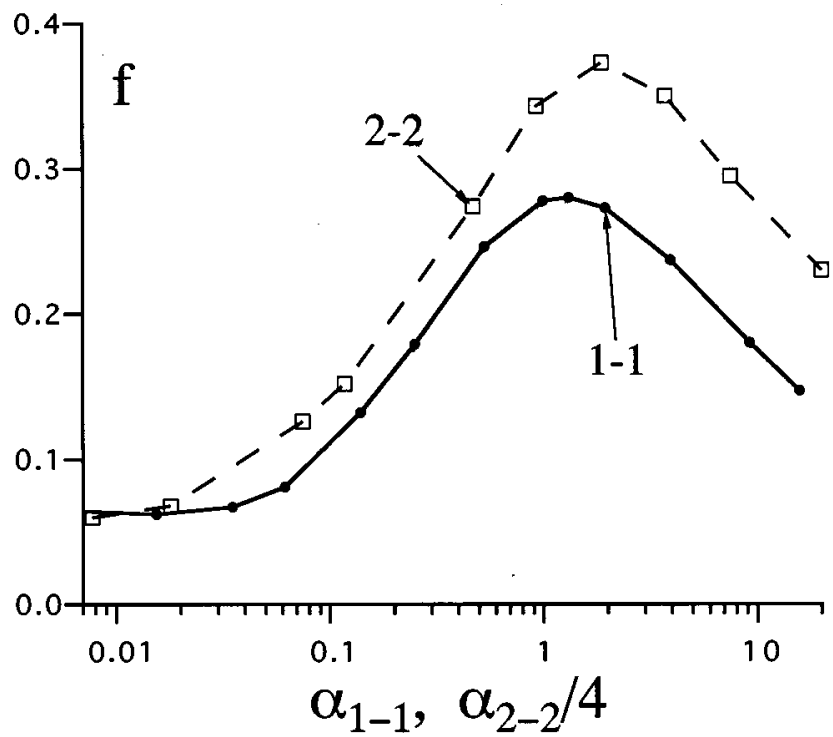

FIG. 5. OBE results for the fraction of trapped helium atoms at a fixed interaction time vs the parameter $\alpha_{1 \rightarrow 1}$ for the $1 \rightarrow 1$ transition and for $\alpha_{2 \rightarrow 2} / 4$ for the $2 \rightarrow 2$ transition. For the $1 \rightarrow 1$ transition the interaction time is $\Theta \omega_{R}=66$ and for the $2 \rightarrow 2$ transition the interaction time is $\Theta \omega_{R}=100$. As explained in the text, the scalings of time and $\alpha$ have been determined by the loss-rate limiting values and the Raman hole width for the two transitions.

$$
f_{0}=\frac{1}{1+\frac{4}{\pi \sqrt{3} \alpha}} .
$$

However, at times $\Theta \geqslant \Theta_{e}$, the trapped fraction decreases, as for helium in Fig. 4, and the maximum reachable trapped fraction is about $0.5 f_{0}-0.7 f_{0}$. We may suppose that at interaction times such that $\Theta \gg \Theta_{e}$ for all the compared atoms, a scaling law of the trapped fraction is valid again, but we have not tested this hypothesis.

The dependence of $f$ on $\alpha$ at a fixed interaction time is reported in Fig. 5. The data of Fig. 5 have been obtained for helium, but because of the scaling law for the trapped fraction, they can be applied to any atom. The $\alpha$ values have been chosen in order to produce a maximum $f$ for an interaction time in helium as typical of the experiments in Refs. $[1,3,4]$. The data, obtained through OBEs with a limited basis of the atomic momentum, correspond to trapped fractions lower than those obtained through the QMC analysis. However, the main aim of Fig. 5 is the comparison with the $F_{g}=2 \rightarrow F_{e}=2$ results obtained through OBE integration. The interaction time was fixed at $\Theta \omega_{R}=66$, corresponding to $\Theta \Gamma^{\prime}=260$ for $\alpha=1$. On increasing $\alpha$, the trapped fraction at a fixed interaction time passes through a maximum. An initial increase of $\alpha$ produces an accumulation of the atoms in the noncoupled state. However, the increase of $\alpha$ decreases $\Theta_{e}$, as it appears from Eq. (24) by using Eq. (19). Such a decrease of $\Theta_{e}$ will produce the relation $\Theta \sim \Theta_{e}$, which is satisfied at a value of $\alpha$ near the maximum of Fig. 5. At larger- $\alpha$ values the VSCPT evolution is within the regime where the Doppler shift decreases the trapping efficiency. 


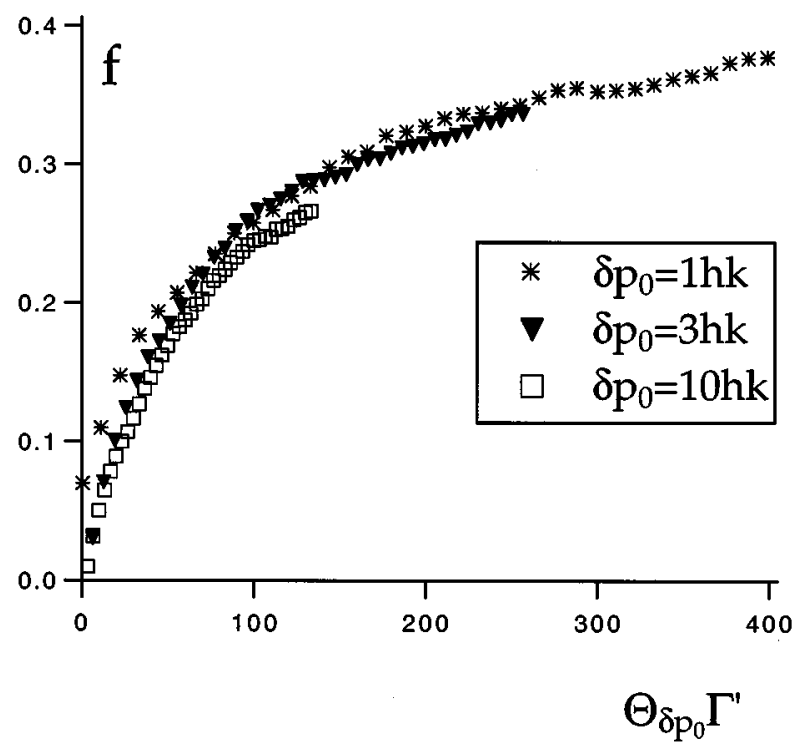

FIG. 6. QMC results for the fraction of trapped helium atoms vs the interaction time $\Theta_{\delta p_{0}} \Gamma^{\prime}$ at $\alpha=1$ for different initial standard deviations of the atomic momentum distribution, i.e., $\delta p_{0}=1 \hbar k$, $3 \hbar k$, and $10 \hbar k$.

Figure 6 reports results for the dependence of the trapped fraction on the initial width of the atomic momentum distribution. The data were obtained from the QMC approach for helium with $\alpha=1$ and initial standard deviations $\delta p_{0}=1 \hbar k$, $3 \hbar k$, and $10 \hbar k$. At the short interaction times shown in Fig. 6 , the VSCPT trapping efficiency depends on $\delta p_{0}$ and decreases if a wider initial atomic distribution is used. On the contrary, in the regime dominated by the Doppler shift, at $\Theta \gg \Theta_{e}$, the trapped fraction becomes independent of the initial width of the atomic momentum distribution, as verified in Ref. [14]. The data of Fig. 6 have been plotted by using a scaling law that we have derived for the dependence of the trapped fraction on the width of the initial atomic distribution. For a large initial width, a long time is required for the atoms to diffuse to the $p \sim \pm \hbar k$ values of the trapped state. Thus we searched for a scaling of the interaction times that would bring together the results obtained at different initial widths. A scaling of the interaction time with the inverse of the initial standard deviation, i.e., $\Theta_{\delta p_{0}}=\Theta /\left(\delta p_{0} / \hbar k\right)$, produces a collapse of the different trapped-fraction dependences into a single curve, as in Fig. 6. An atomic evolution with a constant momentum diffusion would suggest a scaling of the time with $\left(\delta p_{0}\right)^{-2}$, but the scaling applied in Fig. 6 produces a better result.

$$
\text { V. } F_{g}=2 \rightarrow F_{e}=2 \text { TRANSITION }
$$

\section{A. Eigenvalues}

For this transition the eigenvalues are obtained by diagonalizing the effective Hamiltonian $H_{\text {eff }}$ of Eq. (12) on the family $\mathscr{F}(q)$ of Eq. (9b). By eliminating once again a common kinetic-energy term for the three ground states of that family, the $3 \times 3$ matrix of the effective Hamiltonian is

$$
H_{\text {eff }}(q)=\hbar\left(\begin{array}{clc}
4 \omega_{R}+\omega_{|2|}^{\mathrm{LS}}+2 \frac{k q}{m}+R_{11} & R_{12} & 0 \\
R_{21} & \omega_{0}^{\mathrm{LS}} & R_{23} \\
0 & R_{32} & 4 \omega_{R}+\omega_{|2|}^{\mathrm{LS}}-2 \frac{k q}{m}+R_{33}
\end{array}\right)
$$

with the level-shift operators $R_{i j}$ of the resolvent theory reported in the Appendix. The diagonal terms contain the light shift of Eq. (5) produced by an additional $\pi$-polarized laser beam, in order to compensate for the energy mismatch in the leaky case. In effect, the noncoupled state is given by the linear superposition

$$
\begin{aligned}
\left|\mathscr{S}_{\mathrm{NC}}(q)\right\rangle= & \frac{1}{\sqrt{8}}(\sqrt{3}|2,-2 ; q-2 \hbar k\rangle+\sqrt{2}|2,0 ; q\rangle \\
& +\sqrt{3}|2,2 ; q+\hbar k\rangle) .
\end{aligned}
$$

Without light shifts the kinetic-energy difference between the ground states composing $\left|\mathscr{S}_{\mathrm{NC}}(q=0)\right\rangle$ is equal to $4 \omega_{R}$ and only a leaky trapped state is formed [7]. If the additional $\pi$ laser produces light shifts with

$$
\omega_{0}^{\mathrm{LS}}-\omega_{|2|}^{\mathrm{LS}}=4 \omega_{R}
$$

the ground states composing $\left|\mathscr{S}_{\mathrm{NC}}(q=0)\right\rangle$ acquire the same total energy. Thus a nonleaky regime is realized with a stable trapping state [10]. VSCPT results are here presented for both a leaky configuration, i.e., with $\omega_{0}^{\mathrm{LS}}=\omega_{|2|}^{\mathrm{LS}}=0$, and a nonleaky configuration, with Eq. (29) satisfied.

For the $2 \rightarrow 2$ transition we do not have the analytical expressions of the eigenvalues. However, we expect the loss rate $\Gamma_{\mathrm{NC}}(q)$ of the $\left|\mathscr{S}_{\mathrm{NC}}(q)\right\rangle$ state to present a $q$ dependence similar to that of Eq. (21). Figure 7(a) reports results for the loss rate $\Gamma_{\mathrm{NC}}(q)$ as obtained from the diagonalization of the $H_{\text {eff }}$ of Eq. (27) for $\alpha=2$. The case of a nonleaky configuration is reported, so that at $q=0$, the loss rate is exactly zero and a stable trapped state is formed. The loss rates of Fig. 7 are obtained by using the atomic parameters of helium, sodium, and cesium. Only sodium corresponds to a real case, whereas the other ones are imaginary cases. The loss rates for the three atoms are different only for $q \geqslant p_{e}$ because of the Doppler shift. In the absence of any Doppler shift, the function $\Gamma_{\mathrm{NC}}(q)$ would reach a maximum value $2 \Gamma^{\prime} / 3$ at $|q| \rightarrow \infty$, with the pumping rate $\Gamma^{\prime}$ defined in Eq. (16). The region around $q=0$ contains the Raman hole that determines the efficiency of the VSCPT process. At $q \sim 2 \hbar k$ the function 

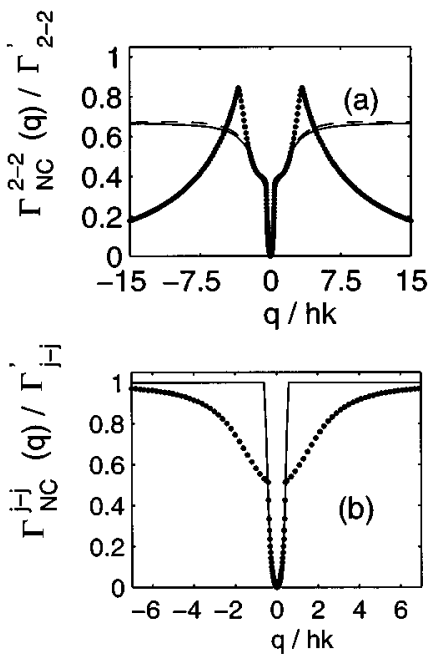

FIG. 7. (a) Loss rate $\Gamma_{\mathrm{NC}}(q) / \Gamma^{\prime}$ plotted vs $q / \hbar k$ for a $2 \rightarrow 2$ transition at $\alpha_{2 \rightarrow 2}=2$ as derived from the effective Hamiltonian. Dots refer to helium, the dashed line to sodium, and the solid line to cesium (b). Plot of $\Gamma_{\mathrm{NC}}^{j \rightarrow j} / \Gamma_{j \rightarrow j}^{\prime}$ vs $q / \hbar k$ for comparing the $1 \rightarrow 1$ and $2 \rightarrow 2$ transitions in cesium. The solid line is for $1 \rightarrow 1$, with $\Gamma_{1 \rightarrow 1}^{\prime}$ $=\Gamma^{\prime}$ at $\alpha_{1 \rightarrow 1}=0.5$. The dotted line is for $2 \rightarrow 2$, with $\Gamma_{2 \rightarrow 2}^{\prime}$ $=\left(2 \Gamma^{\prime} / 3\right)$ and $\alpha_{2 \rightarrow 2}=2$.

$\Gamma_{\mathrm{NC}}(q)$ presents a discontinuity in its derivative, because for each $q$ value we report the $\Gamma_{\mathrm{NC}}(q)$ value of the state with the smallest loss rate. As a function of $q$, different linear combinations of states or, more precisely, states associated with a different energy band [23] may have the smallest loss rate.

Figure $7(b)$ is the basis of a scaling law to compare the trapped fraction for the $1 \rightarrow 1$ and $2 \rightarrow 2$ transitions. Those trapped fractions may be compared if the interaction time is measured in units of the pumping time and if the Raman hole area is the same. For comparing the $1 \rightarrow 1$ and $2 \rightarrow 2$ transitions, the interaction time should be measured in units of $1 / \Gamma^{\prime}$ and $3 / 2 \Gamma^{\prime}$, respectively, which represent the time scales of the atomic evolutions outside the Raman hole. For comparing the Raman holes of the two transitions, we notice that $\alpha$ is inversely proportional to the recoil frequency $\omega_{R}$ and, for the $2 \rightarrow 2$ case, the $\left|\mathscr{S}_{\mathrm{NC}}(0)\right\rangle$ state of Eq. (28) contains a kinetic evolution at the $4 \omega_{R}$ frequency. Thus, in order to obtain the same Raman hole of the $1 \rightarrow 1$ transition, the $\alpha$ parameter of the $2 \rightarrow 2$ transition should be increased by a factor 4. In Fig. 7(b) the loss rates $\Gamma_{\mathrm{NC}}^{i \rightarrow i}(q) / \Gamma_{i \rightarrow i}^{\prime}(i=1,2)$ with $\Gamma_{1 \rightarrow 1}^{\prime}=\Gamma^{\prime}$ and $\Gamma_{2 \rightarrow 2}^{\prime}=2 \Gamma^{\prime} / 3$ are compared as function of $q$ for $\alpha_{1 \rightarrow 1}=0.5$ and $\alpha_{2 \rightarrow 2}=2$. Having applied those scalings, the central part of the Raman hole in the two transitions presents good agreement.

\section{B. Trapped fraction}

The density-matrix time evolution has been examined through the OBE numerical solution and all the results here presented are based on an initial atomic momentum Gaussian distribution with standard deviation $\delta p_{0}=\hbar k$. Figure 8 reports results for the dependence of the trapped fraction $f$ on the interaction time $\Theta \Gamma^{\prime}$ for the case of leaky and nonleaky transitions at $\alpha=2$. Parameters for four different atoms have been used: helium, sodium, rubidium, and cesium, with he-

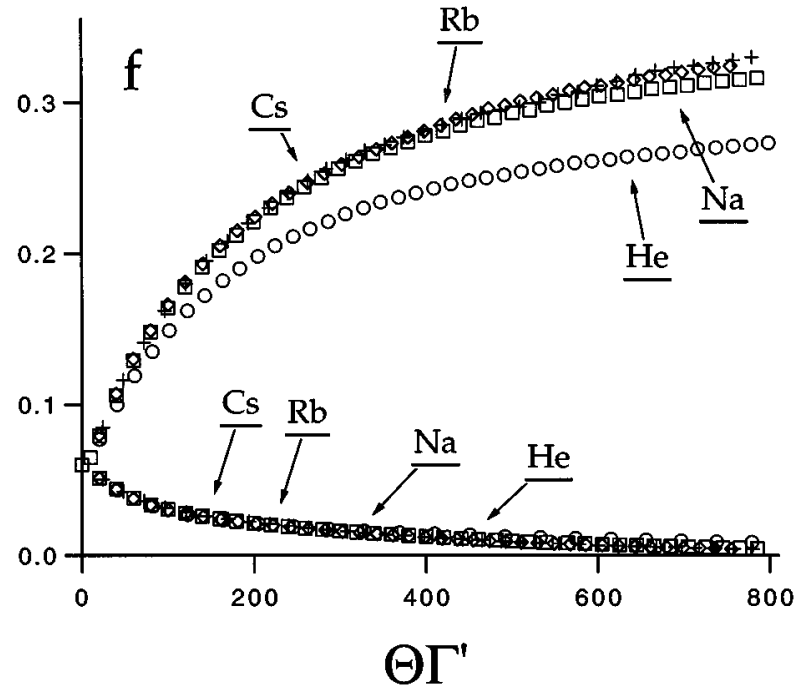

FIG. 8. OBE results for the fraction of trapped atoms vs interaction time $\Theta \Gamma^{\prime}$ at $\alpha=2$ for the $2 \rightarrow 2$ transition, using parameters of helium, sodium, rubidium, and cesium atoms. The data with a larger trapped fraction correspond to the nonleaky configuration, the others to the leaky configuration. As for the case of the $1 \rightarrow 1$ transition, the trapped fraction does not depend on the atomic parameters.

lium and cesium imaginary atomic transitions. It may be noticed that the scaling law provides a very good description for the time dependences of both the leaky and nonleaky cases.

Figure 5 reports the dependence of the helium trapped fraction on the $\alpha$ parameter at a fixed interaction time $\Theta$ for a comparison between the $2 \rightarrow 2$ and $1 \rightarrow 1$ transitions. The comparison between the two transitions is based on the scaling laws discussed in Sec. IV for the interaction time and the $\alpha$ parameter. The results should be compared for $\alpha$ parameters scaled by a factor 4 in order to have the same Raman hole, i.e., $\alpha_{2 \rightarrow 2}=4 \alpha_{1 \rightarrow 1}$. For $2 \rightarrow 2$, the interaction time is measured in units of $\Gamma_{2 \rightarrow 2}^{\prime}=2 \Gamma^{\prime} / 3$, whereas for $1 \rightarrow 1$, it is measured in units of $\Gamma^{\prime}$. Thus the trapped fractions of Fig. 5 have been determined at time $\Theta=100 / \omega_{R}$ for the $2 \rightarrow 2$ transition and at time $\Theta=66 / \omega_{R}$ for the $1 \rightarrow 1$ transition. The scaling leads to very good agreement between the trapped fractions on the two transitions. It should be noted that without applying that scaling, the trapped fractions of the $2 \rightarrow 2$ and $1 \rightarrow 1$ transitions would appear uncorrelated. It is surprising to note that the maximum in the $2 \rightarrow 2$ trapped fraction is larger than in the $1 \rightarrow 1$ one. However, the plots of the $2 \rightarrow 2$ loss rate of Fig. 7 present a large "hole" area outside the proper Raman hole area that is not present for the $1 \rightarrow 1$ transition. That area may produce the larger efficiency of the $2 \rightarrow 2$ VSCPT process. Also Wu, Holland, and Foot [12], in their comparison between the VSCPT efficiency on the $2 \rightarrow 2$ and $1 \rightarrow 1$ transitions, have shown that for a fixed Rabi frequency, the $2 \rightarrow 2$ VSCPT is more efficient than the $1 \rightarrow 1$ one. Figure 5 provides a complete description for the VSCPT on the two transitions with a comparison of their efficiencies at different Rabi frequencies.

In order to provide an additional confirmation of the scaling law between the two transitions, we have examined the width of the atomic momentum distribution associated with 


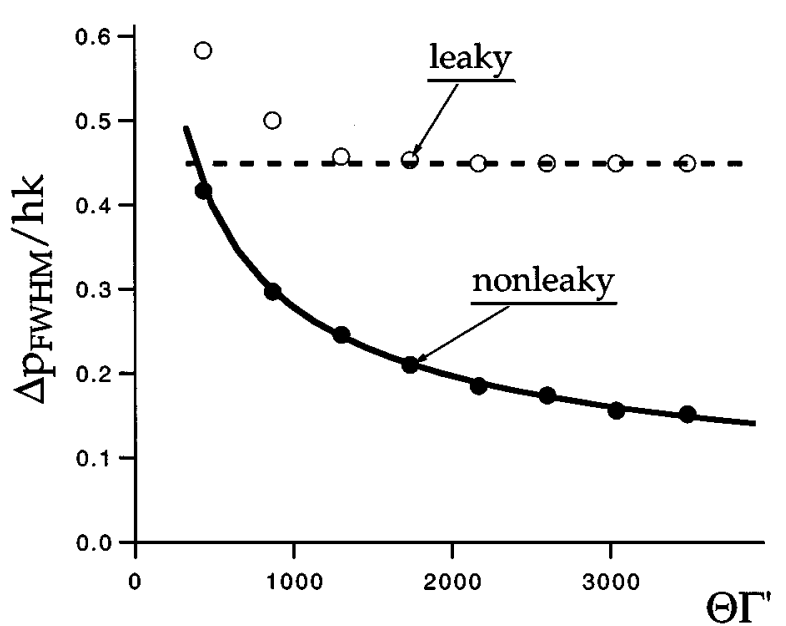

FIG. 9. FWHM $\Delta p_{\text {FWHM }}(\Theta) / \hbar k$ of the atomic momentum distribution vs the interaction time $\Theta \Gamma^{\prime}$, for the $2 \rightarrow 2$ transition using the cesium atomic parameters, at $\Omega=0.16 \Gamma$ corresponding to $\alpha=8$ and the initial Gaussian distribution with standard deviation $\delta p_{0}=\hbar k$. Data for the two cases of a leaky (open dots) and a nonleaky (closed dots) configuration are reported. The dotted line defines the limiting value of $\Delta p_{\text {FWHM }}=0.45 \hbar k$ for the leaky case. The continuous line represents the $\Delta p_{\mathrm{FWHM}}=50\left(\Theta \Gamma^{\prime}\right)^{-1 / 2}$ fit of the nonleaky data.

the VSCPT process, measured at $p= \pm \hbar k$ for the $1 \rightarrow 1$ transition and at $p=0, \pm 2 \hbar k$ for the $2 \rightarrow 2$ transition. For the $1 \rightarrow 1$ transition, the $\Theta^{-1 / 2}$ dependence of the width on the interaction time $\Theta$ has been characterized by Alekseev and Kyrola [24] and by Mauri and Arimondo [25]. In particular, Ref. [24] has pointed out that the momentum distribution is described by a Lorentzian line shape, so that its width is described through the full width at half maximum $\Delta p_{\text {FWHM }}(\Theta)$. For the $2 \longrightarrow 2$ atomic transition with cesium parameters, at $\alpha_{2 \rightarrow 2}=8$ we have determined $\Delta p_{\text {FWHM }}$ versus $\Theta$, for the cases of leaky and nonleaky transitions, with results shown in Fig. 9. We have verified that the momentum distributions are well described by Lorentzian line shapes. For the leaky case, the momentum width reaches a limiting value $\Delta p_{\text {FWHM }}=0.45 \hbar k$, within $20 \%$ of the value given by an analytical relation derived in Ref. [26]. For the nonleaky case the data are well fitted by the equation $\Delta p_{\mathrm{FWHM}}=50\left(\Theta \Gamma^{\prime}\right)^{-1 / 2}$, as shown by the continuous line drawn through the data. By comparing this relation with the $\Delta p_{\text {FWHM }}(\Theta)$ dependences reported in Refs. [24] and [25] for the $1 \rightarrow 1$ transition, taking into account the scaling law of $\Gamma_{j \rightarrow j}^{\prime}$, we find that a similar dependence would be obtained at $\alpha_{1 \rightarrow 1}=2.3 \pm 0.3$ for the $1 \rightarrow 1$ transition, instead of $\alpha_{2 \rightarrow 2}=8$. Thus the scaling of $\alpha$ by a factor 4 is confirmed also by the dependence of the momentum distribution width on the interaction time.

We have investigated the dependence of the trapped fraction on the interaction time in the presence of a laser detuning $\delta_{L} \neq 0$. In effect, for the $2 \rightarrow 2$ transition for a detuning different from zero, a polarization-gradient cooling force is acting on the atoms, as discussed in Refs. [27], and that force may increase the trapping efficiency. In our numerical simulations for $\delta p_{0}=\hbar k$ and different laser detunings, we have not found a significant modification for the trapped-fraction dependence on $\Theta \Gamma^{\prime}$, when the modification of the pumping rate by the laser detuning is taken into account. It may be supposed that owing to the quite narrow initial momentum distribution, the role of the polarization-gradient cooling is limited. Thus a more correct comparison should be performed with larger initial momentum distributions, as recently reported in Ref. [28].

\section{CONCLUSION}

The aim of the present work is to determine relations connecting the principal features of VSCPT laser cooling on different $F_{g} \rightarrow F_{e}$ atomic transitions. Those relations have been derived from a comparison between the VSCPT of the $1 \rightarrow 1$ and $2 \rightarrow 2$ transitions for different atomic parameters. Scaling laws have been derived for the dependence of the fraction of trapped atoms on the interaction time and the Rabi frequency. A relevant scaling parameter $\alpha$ has been defined as the square of the Rabi frequency divided by the atomic spontaneous-emission decay and the atomic recoil frequency. As a consequence of the dependence of the trapped fraction on $\alpha$ only, to prepare (for instance, in the VSCPT cooled state) a given fraction of heavy atoms having a small recoil frequency, a decrease of the Rabi frequency is required, keeping $\alpha$ constant. Owing to the good validity of the scaling laws for the two investigated atomic transitions, we are confident that the scaling laws may be applied to atomic transitions having a larger atomic momentum, for instance, the real $F_{g}=4 \rightarrow F_{e}=4$ transition of the cesium resonant line. The scaling law predicts that the maximum efficiency in the cesium VSCPT will be reached at Rabi frequencies much smaller than those used in the helium experiments.

The application of these scaling laws to other laser schemes involving VSCPT may be considered. We have verified that the bichromatic VSCPT scheme of Doery et al. [11] is completely equivalent to a nonleaky VSCPT scheme on the $2 \rightarrow 2$ transition. Thus the same trapped fraction is realized in the two schemes and, as a consequence, the scaling laws derived for the dependence of the VSCPT trapped fraction on the interaction time and the Rabi frequency are valid also for the bichromatic VSCPT scheme.

The application of a standing wave on each optical transition of the $1 \rightarrow 1$ scheme produces a VSCPT subrecoil cooling combined with polarization-gradient laser cooling, as discussed in Refs. [15-18]. Those authors have shown that for a given interaction time, an increase of the trapped fraction is achieved by alternating periods of pure VSCPT, at zero detuning, with periods of polarization-gradient cooling, at a laser detuning different from zero. For the pure VSCPT, our scaling laws apply. For the polarization-gradient laser cooling, an investigation of the scaling laws has been performed by Castin et al. [29]. These authors have shown that polarization-gradient cooling is described by two independent parameters $\delta_{L} / \Gamma$ and $\Gamma^{\prime} \delta_{L} / \Gamma \omega_{R}$. Thus, in polarizationgradient cooling, the laser detuning plays an important role and within the search for scaling laws in the cooling combination of VSCPT and a polarization gradient, the laser detuning should be considered as a separate parameter, in addition to $\alpha$. In fact, the second parameter introduced by Castin et al. is a combination of $\alpha$ and $\delta_{L}$. The existence of scaling laws may be examined also for Doppler cooling. The 
Doppler cooling process is characterized by a temperature proportional to the spontaneous-emission rate, with its efficiency given by the atoms that have reached a kinetic-energy range around that temperature. The interaction time for reaching that efficiency is scaled once again with the pumping rate, proportional to the number of scattered spontaneous-emission photons. The dependence on the Rabi frequency appears in the Doppler cooling equations through the $\alpha$ parameter when an adiabatic elimination of the excited state is performed. Thus the same parameters of the VSCPT scaling laws determine the Doppler cooling efficiency.

\section{ACKNOWLEDGMENTS}

G.M. thanks G. Hillenbrand for the useful discussions on the resolvent theory and E.A. thanks G. Grynberg and P. Marte for helpful comments. The work by N.L. was partially supported by the Austrian Science Foundation under Project No. S 6508 and his visit at the Dipartimento di Fisica dell'Università di Pisa was supported by the Ministero degli Affari Esteri d'Italia, in the framework of the Austria-Italy scientific collaboration.

\section{APPENDIX}

The expressions of the level-shift operators for the $F_{g}=2 \rightarrow F_{e}=2$ transition appearing in Eq. (27) are

$$
\begin{aligned}
& R_{11}=\frac{1}{4} \frac{\Omega^{2} / 3}{\delta_{0}-\omega_{R}+k q / m+i \Gamma / 2}, \\
& R_{12}=\frac{1}{4} \frac{\Omega^{2} / \sqrt{6}}{\delta_{2}-\omega_{R}-k q / m+i \Gamma / 2}, \\
& R_{21}=\frac{1}{4} \frac{\Omega^{2} / \sqrt{6}}{\delta_{0}-\omega_{R}+k q / m+i \Gamma / 2},
\end{aligned}
$$

$$
R_{22}=\frac{1}{4} \frac{\Omega^{2} / 2}{\delta_{2}-\omega_{R}-k q / m+i \Gamma / 2}+\frac{1}{4} \frac{\Omega^{2} / 2}{\delta_{2}-\omega_{R}+k q / m+i \Gamma / 2},
$$

$$
\begin{aligned}
& R_{23}=\frac{1}{4} \frac{\Omega^{2} / \sqrt{6}}{\delta_{0}-\omega_{R}-k q / m+i \Gamma / 2}, \\
& R_{32}=\frac{1}{4} \frac{\Omega^{2} / \sqrt{6}}{\delta_{2}-\omega_{R}+k q / m+i \Gamma / 2},
\end{aligned}
$$

$$
R_{33}=\frac{1}{4} \frac{\Omega^{2} / 3}{\delta_{0}-\omega_{R}-k q / m+i \Gamma / 2},
$$

where $\delta_{2}=\omega_{L}-\omega_{0}+4 \omega_{R}+\omega_{|2|}^{\mathrm{LS}}$ and $\delta_{0}=\omega_{L}-\omega_{0}+\omega_{0}^{\mathrm{LS}}$.
[1] A. Aspect, E. Arimondo, R. Kaiser, N. Vansteenkiste, and C. Cohen-Tannoudji, Phys. Rev. Lett. 61, 826 (1988).

[2] A. Aspect, E. Arimondo, R. Kaiser, N. Vansteenkiste, and C. Cohen-Tannoudji, J. Opt. Soc. Am. B 6, 2112 (1989).

[3] F. Bardou, B. Saubamea, J. Lawall, K. Shimizu, O. Émile, C. Westbrook, A. Aspect, and C. Cohen-Tannoudji, C. R. Acad. Sci. Paris, Série II 318, 877 (1994).

[4] M. R. Doery, M. T. Widmer, M. J. Bellanca, W. F. Buell, T. H. Bergeman, H. Metcalf, and E. J. D. Vredenbregt, Phys. Rev. A 52, 2295 (1995).

[5] T. Esslinger, F. Sandler, M. Weidemüller, A. Hemmerich, and T. W. Hänsch, Phys. Rev. Lett. (to be published).

[6] J. Lawall, F. Bardou, B. Saubamea, K. Shimizu, M. Leduc, A. Aspect, and C. Cohen-Tannoudji, Phys. Rev. Lett. 73, 1915 (1994).

[7] F. Papoff, F. Mauri, and E. Arimondo, J. Opt. Soc. Am. B 9, 321 (1992).

[8] M. A. Ol'shaniî, J. Phys. B 24, L583 (1991).

[9] M. A. Ol'shanii, Opt. Spectrosc. 76, 174 (1994).

[10] C. Foot, H. Wu, E. Arimondo, and G. Morigi, J. Phys. (France) II 4, 1913 (1994).

[11] M. R. Doery, R. Gupta, T. Bergeman, H. Metcalf, and E. J. D. Vredenbregt, Phys. Rev. 51, 2334 (1995).

[12] H. Wu, M. J. Holland, and C. Foot, J. Phys. B 28, 5025 (1995).

[13] F. Bardou, J. P. Bouchaud, O. Émile, A. Aspect, and C. CohenTannoudji, Phys. Rev. Lett. 72, 203 (1994).

[14] A. Fioretti, B. Zambon, and E. Arimondo, Quant. Semiclass. Opt. 7, 751 (1995).
[15] M. S. Shahriar, P. R. Hemmer, M. G. Prentiss, P. Marte, J. Mervis, D. P. Katz, N. P. Bigelow, and T. Cai, Phys. Rev. A 48, R4035 (1993).

[16] P. Marte, R. Dum, R. Täeb, P. Zoller, M. S. Shahriar, and M. Prentiss, Phys. Rev. A 49, 4826 (1994).

[17] M. Weidemüller, T. Esslinger, M. A. Ol'shanii, A. Hemmerich, and T. W. Hänsch, Europhys. Lett. 27, 109 (1994).

[18] R. Dum (unpublished).

[19] P. R. Berman, Phys. Rev. A 43, 1470 (1991); P. R. Berman, G. Rogers, and B. Dubetsky, ibid. 48, 1506 (1993); P. Marte, R. Dum, R. Taieb, and P. Zoller, ibid. 47, 1379 (1993); M. R. Doery, E. J. D. Vredenbregt, and T. Bergeman, ibid. 51, 4881 (1995).

[20] K. Mølmer, Y. Castin, and J. Dalibard, J. Opt. Soc. Am. B 10, 524 (1993).

[21] C. Cohen-Tannoudji, J. Dupont-Roc, and G. Grynberg, AtomPhoton Interactions (Wiley, New York, 1992); A. Bohm, Quantum Mechanics, Foundations and Applications (Springer, New York, 1993).

[22] J. Dalibard and C. Cohen-Tannoudji, J. Opt. Soc. Am. B 6, 2023 (1989).

[23] Y. Castin and J. Dalibard, Europhys. Lett. 14, 761 (1991).

[24] V. A. Alekseev and D. D. Kyrola, Laser Phys. 2, 781 (1992); Opt. Commun 95, 319 (1993).

[25] F. Mauri and E. Arimondo, Appl. Phys. B 54, 420 (1992).

[26] A. V. Taichenachev, A. M. Tumaikin, and V. I. Yudin, Laser Phys. 2, 575 (1992). 
[27] G. Nienhuis, P. Van der Straten, and S.-Q. Shang, Phys. Rev. A 44, 42 (1991); A. M. Steane, G. Hillenbrand, and C. Foot, J. Phys. B 25, 4721 (1992); J. Werner, H. Wallis, G. Hillenbrand, and A. Steane, ibid. 26, 3063 (1993).
[28] H. Wu, G. Hillenbrand, G. Morigi, and C. Foot, J. Phys. B 28, 5269 (1995).

[29] Y. Castin, K. Berg-Sørensen, J. Dalibard, and K. Mølmer, Phys. Rev. A 50, 5092 (1994). 